\title{
Nuclear model developments in FLUKA for present and future applications
}

\author{
Francesco Cerutti ${ }^{1}$, Anton Empl ${ }^{2}$, Anatoli Fedynitch ${ }^{3}$, Alfredo Ferrari ${ }^{1, a}$, Ruben Garcia Alia ${ }^{1}$, Paola R. Sala ${ }^{1,4}$, \\ George Smirnov ${ }^{1}$, and Vasilis Vlachoudis ${ }^{1}$ \\ 1 CERN, 1211 Genève 23, Switzerland \\ 2 Department of Physics, University of Houston, Houston, TX 77204, USA \\ 3 DESY, Platanenallee 6, 15738 Zeuthen, Germany \\ ${ }^{4}$ INFN, Via Celoria 16, 20133 Milan, Italy
}

\begin{abstract}
The FLUKA code [1-3] is used in research laboratories all around the world for challenging applications spanning a very wide range of energies, projectiles and targets. FLUKA is also extensively used for in hadrontherapy research studies and clinical planning systems. In this paper some of the recent developments in the FLUKA nuclear physics models of relevance for very different application fields including medical physics are presented. A few examples are shown demonstrating the effectiveness of the upgraded code.
\end{abstract}

\section{Introduction}

Studies carried out with FLUKA are often at the heart of the design of projects or experiments in domains where little or no data are available, therefore requiring particular attention to predictive power. In particular, the high energy beams which became recently available at the CERN LHC $(6.5+6.5 \mathrm{TeV})$ and the even higher energy ones $(50+50 \mathrm{TeV})$ envisaged for the future Future Circular Collider project required a careful analysis and improvement of the high energy models embedded into FLUKA in order to make physics driven extrapolations into uncharted territory. At the opposite end of the energy range, the widespread use of FLUKA in hadrontherapy research studies and clinical planning systems demands extreme accuracy. Predictions on 3D dose distributions in patients are indeed expected to be accurate within a couple of percent, and reliable informations about fragment composition and secondary radiations are required for biological effectiveness assessments and online monitoring, respectively.

\section{Dpmjet-III improvements}

LHC successfully ran up to $13 \mathrm{TeV} \mathrm{cms}$ with record luminosity providing, as a by-product, plenty of data of relevance for machine protection, shielding, and other applications.

Comparisons with p-p (and $\mathrm{Pb}-\mathrm{Pb}$ ) event generators have shown areas where improvements are required, with the exception of Electro Magnetic Dissociation (EMD) results for $\mathrm{Pb}-\mathrm{Pb}$ interactions, where predictions were remarkably accurate. Model improvements were critical, both for the safe operation of the machine at $6.5+6.5 \mathrm{TeV}$, for the forthcoming High Luminosity LHC upgrade (HL-

a e-mail: alfredo.ferrari@cern.ch
LHC), and for the (hypothetical) Future Circular Collider, a $100 \mathrm{~km}$ ring hosting a proton-proton $(50+50 \mathrm{TeV})$ collider and an electron-positron (up to $175+175 \mathrm{GeV}$ ) one.

For heavy ion interactions above $5 \mathrm{GeV} / \mathrm{n}$, as well as for hadron-hadron and hadron-nucleus interactions above $20 \mathrm{TeV}$ FLUKA interfaces to DPMJET-III [4], the nuclear framework around the PHOJET [5,6] event generator for hadron-hadron, photon-hadron and photonphoton collisions. Initial comparisons with LHC data for pp collisions at $\sqrt{s}=7 \mathrm{TeV}$ showed some discrepancies which were addressed in a comprehensive review and update of the PHOJET models described elsewhere [7]. The modified code compares well with a wide variety of data taken at LHC, two examples being presented in Figs. 1,2.

\section{New features in electro magnetic dissociation}

EMD in FLUKA has been already tested and applied at LHC energies with sound results [9]. The model has now been improved, including the E2 multipolarity, nuclear finite size and higher order effects, which are very important at low energy and for $e^{ \pm}$and $\mu$. FLUKA EMD has been extended to handle muon-induced photonuclear interactions below the $\gamma+N \rightarrow \pi+X$ threshold, and is now able to simulate electronuclear interactions. The same formalism applies also to Deuteron Coulomb dissociation. The EMD cross section can be expressed as a sum over multipolarity $i$ of the convolution of the number of emitted (virtual) photons $n_{i}$ with the photon-nucleus cross section $\sigma_{i \gamma A}$.

$$
\sigma_{E M D} \approx \sum_{i} \int_{E_{\min }}^{E_{\max }} \sigma_{i \gamma A}\left(E_{\gamma}\right) n_{i}\left(E_{\gamma}\right) \frac{\mathrm{d} E_{\gamma}}{E_{\gamma}}
$$

(C) The Authors, published by EDP Sciences. This is an Open Access article distributed under the terms of the Creative Commons Attribution License 4.0 (http://creativecommons.org/licenses/by/4.0/). 


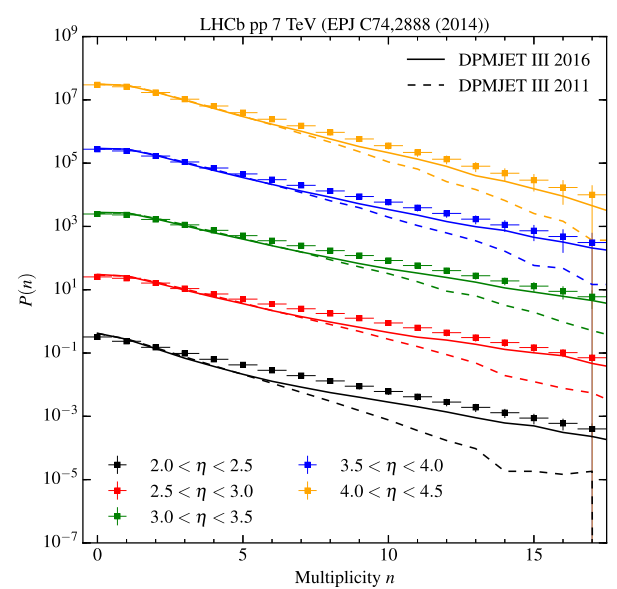

Figure 1. Multiplicity distribution of charged particles for $\mathrm{pp}$ collision at $\sqrt{s}=7 \mathrm{TeV}$ for various intervals of pseudorapidity, as measured (symbols) by the LHCb experiment [8], and as simulated by DPMJET-III before (dashed lines) and after (solid lines) the recent improvements.

It can be shown that the dominant components are E1 and E2, with E2 being (for ions) important mostly at low energies, while M1 is always negligible, so that Eq. (1) becomes:

$$
\begin{aligned}
\sigma_{E M D} \approx \int_{E_{\min }}^{E_{\max }} & {\left[\sigma_{E 1 \gamma A}\left(E_{\gamma}\right) n_{E 1}\left(E_{\gamma}\right)+\right.} \\
& \left.\sigma_{E 2 \gamma A}\left(E_{\gamma}\right) n_{E 2}\left(E_{\gamma}\right)\right] \frac{\mathrm{d} E_{\gamma}}{E_{\gamma}}
\end{aligned}
$$

Introducing the adiabacity parameter $\xi$,

$$
\xi=\frac{\omega b}{\hbar \beta \gamma} \equiv \frac{\omega}{\omega_{\max }}
$$

the equivalent (virtual) photon number can be expressed as [10]:

$$
\begin{aligned}
n_{E 1}\left(E_{\gamma}\right)= & \frac{2 \alpha Z^{2}}{\pi \beta^{2}}\left[\xi K_{0}(\xi) K_{1}(\xi)-\frac{1}{2} \xi^{2} \beta^{2}\left(K_{1}^{2}(\xi)\right.\right. \\
& \left.\left.-K_{0}^{2}(\xi)\right)\right] \\
n_{E 2}\left(E_{\gamma}\right)= & \frac{2 \alpha Z^{2}}{\pi \beta^{4}}\left[2\left(1-\beta^{2}\right) K_{1}^{2}(\xi)+\xi\left(2-\beta^{2}\right)^{2}\right. \\
& \left.K_{0}(\xi) K_{1}(\xi)-\frac{1}{2} \xi^{2} \beta^{4}\left(K_{1}^{2}(\xi)-K_{0}^{2}(\xi)\right)\right]
\end{aligned}
$$

where $K$ denotes the modified Bessel functions of the second kind, $\omega_{\max }=\frac{\hbar \beta \gamma}{b_{\min }}$ and the minimum impact parameter depends on the emitting particle:

$$
b_{\text {min }}^{\text {ion }}=R_{A B}+\delta_{\text {Ruth }} \quad b_{\text {min }}^{\text {lepton }}=\lambda_{c}=\frac{\hbar}{\beta \gamma m_{\text {lepton }}}
$$

It is worthwhile to note in Eq. (4) the different $\beta$ dependence of the two multipolarities, which enhances the importance of the E2 component at low energies. As mentioned before, the previous implementation of EMD in FLUKA did not include E2.

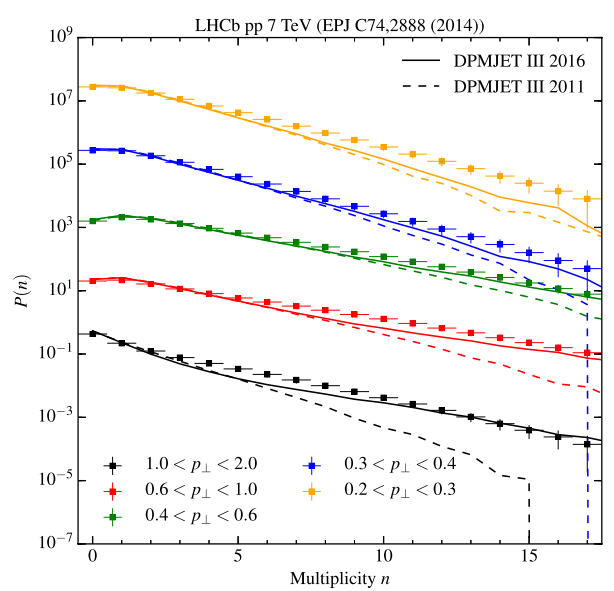

Figure 2. Multiplicity distribution of charged particles for pp collision at $\sqrt{s}=7 \mathrm{TeV}$ for various intervals of transverse momentum, integrated over the pseudorapidity range $\eta=2-$ 4.5 , as measured (symbols) by the LHCb experiment [8], and as simulated by DPMJET-III before (dashed lines) and after (solid lines) the recent improvements.

Relations 4 and 5 are partially based on the first Born approximation. Many papers discuss the E1, M1 and E2 virtual photon spectra emitted by $e^{ \pm}$including both higher Born approximations and the effect of nuclear/charge finite size (see for instance [11-14]). The numerical calculations of $[13,14]$ have been used to derive the corrections newly implemented in FLUKA, through modification of $b_{\text {min }}^{\text {lepton }}$ with respect to the standard formulation of Eq. (5). Accounting for 2 nd Born terms and nuclear finite size effects results in a reduction of the E2 component, a reduction which is larger the higher the nuclear charge $Z$. For $\omega$ close to the emitting particle energy, and for high energies, the first order approach maintains its validity. An alternative derivation, the Equivalent Photon Approximation (EPA) [15], which is often used to describe virtual photon spectra by high energy electrons, agrees with the first order formulation for high energy electrons and high $\omega$, but underestimates the production at low $\omega$.

The E2 contribution is important in order to reproduce EMD data for ions at energies of a few hundreds $\mathrm{MeV} / \mathrm{n}$. For instance, FLUKA predicts that E2 accounts for $25 \%$ of the total EMD cross section for ${ }^{238} U$ on ${ }^{238} U$ at $120 \mathrm{MeV} / \mathrm{n}$, a value in agreement with other theoretical predictions [17] and consistent with experimental total cross sections.

The FLUKA EMD model has been extended to handle electronuclear interactions. Figure 3 shows the good performance of the model in reproducing the electronuclear cross section for $\left(e^{-}, n\right)$ on ${ }^{181} \mathrm{Ta}$ for electron energies in the $10-30 \mathrm{MeV}$ energy interval. Finally, Fig. 4 demonstrates that electrofission on ${ }^{238} \mathrm{U}$ is very well reproduced, both for electron and positron beams. Muon induced photonuclear interactions were already modeled in FLUKA, but only for virtual photon energies above the threshold for pion production on nucleons. An application sensitive to details of the reaction modeling is the evaluation of muon induced background in underground experiments. Comparison [21] of data from the Borexino [22] and Kamland [23] experiments on muon-produced radioisotopes in a liquid scintillator is shown in Fig. 5. The average energy of muons reaching 


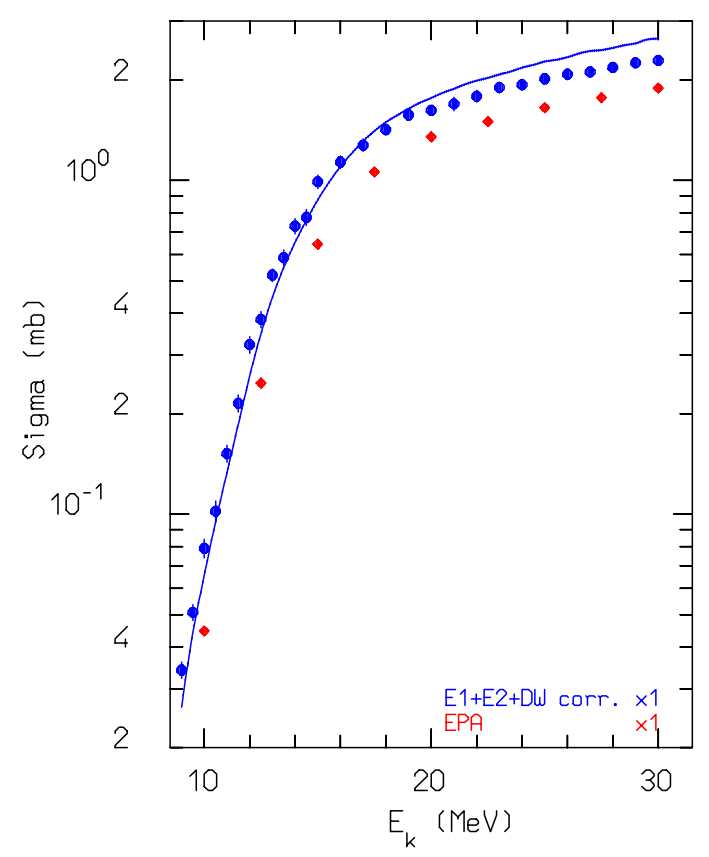

Figure 3. Electronuclear cross section for ${ }^{181} \mathrm{Ta}\left(e^{-}, n\right)^{180} \mathrm{Ta}$ as a function of $e^{-}$energy in the few-tens of $\mathrm{MeV}$ range. Blue dots are experimental data from [16], red dots are FLUKA simulations with EPA, the blue line are FLUKA results with the full approach described in the present paper.

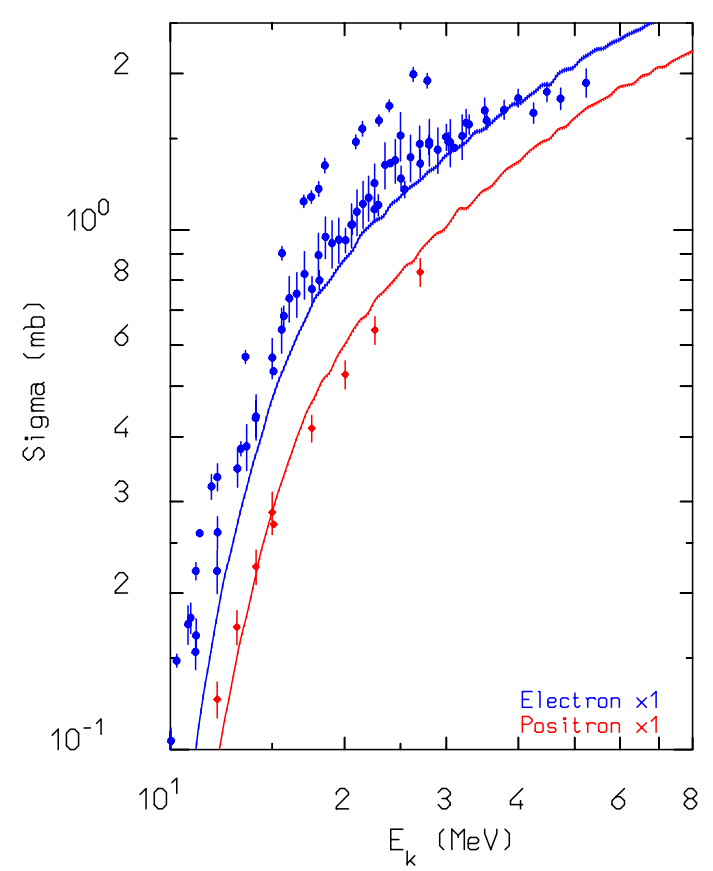

Figure 4. Fission cross section for electrons (blue) and positrons (red) on ${ }^{238} \mathrm{U}$ as a function of electron energy. Symbols: experimental data from [18-20], lines: FLUKA EMD.

the Gran Sasso laboratory is $283 \mathrm{GeV}$. The improvements to the EMD model described above have brought the FLUKA results in excellent agreement with experimental data [21] about total neutron production and neutron multiplicities, and, together with the improvements in Fermi break-up described in [2] they helped in bringing in agreement with the experiment the production of ${ }^{11} C$ which was before underestimated by a factor $\approx 2$ by all models.

\section{Improvements in the BME and $\mathrm{rQMD}$ nucleus-nucleus event generators}

Nucleus-nucleus interactions in FLUKA are treated by an extensively modified version [24] of RQMD-2.4 [25,26] for ion energies between $150 \mathrm{MeV} / \mathrm{n}$ and $5 \mathrm{GeV} / \mathrm{n}$ and by the BME model [27] for energies below $100 \mathrm{MeV} / \mathrm{n}$. Between 100 and $150 \mathrm{MeV} / \mathrm{n} \mathrm{BME}$ is progressively phased out and RQMD phased in, both models being somewhat at the limit of their ranges. This energy range is of great importance for hadrontherapy applications where FLUKA has a leading role in Europe [28], hence an effort has been made in order to improve both models and their transition regime by:

- implementing a preequilibrium stage in RQMD, using the FLUKA standard preequilibrium model;

- making RQMD aware of exact isotope-specific binding energies instead of average ones as well as other isotope specific refinements;

- supplementing the precomputed (complete fusion) preequilibrium configurations database of BME with an interface to the FLUKA preequilibrium in order to be able to treat whichever isotope/production mechanism combinations, plus some general improvements.

The previous version of RQMD was already performing reasonably well at energies well above the transition, as demonstrated in Fig. 6 for Nitrogen ions on Carbon at $400 \mathrm{MeV} / \mathrm{n}$. The improvements not only substantially ameliorated RQMD performances in the transition energy region, but they have also marginally improved the already good agreement at higher energies as shown in Fig. 7 where the same comparison with $400 \mathrm{MeV} / \mathrm{n}$ data is repeated. Figures 8 and 9 present the neutron double differential production cross sections for Carbon ions on Carbon at $135 \mathrm{MeV} / \mathrm{n}$, as computed with the improved $\mathrm{BME}$ and $\mathrm{RQMD}$. As it is apparent from those figures, the consistency between the two codes and the agreement with the experimental data is now fairly satisfactory.

\section{Pointwise, fully correlated cross sections for neutrons on Carbon}

Using pointwise cross sections and fully correlated final state products for neutrons below $20 \mathrm{MeV}$ is a long term goal of FLUKA (and not only, see for example [31]). The main issue is that evaluated data files are hopelessly inclusive, and hence do not allow to model exclusive reaction channels with closed kinematics, apart from simple cases (e.g. elastic scattering). As a step along this direction, fully correlated pointwise cross sections and interactions have been implemented in FLUKA for: ${ }^{1} \mathrm{H},{ }^{2} \mathrm{H},{ }^{3} \mathrm{He},{ }^{4} \mathrm{He},{ }^{6} \mathrm{Li},{ }^{10} \mathrm{~B}$ (only partially), ${ }^{12 / n a t} \mathrm{C}$, the goal being to start from isotopes of relevance for neutron detector simulations. The most recent addition are the carbon cross sections and interactions. ${ }^{12 / n a t} \mathrm{C}$ pointwise/correlated interactions have been generated starting from ENDF/B-VII.1, with corrections for $(n, \alpha)$ taken from EAF2010, adding model/experimental extra informations where required for correlations, and changing some of the cross sections (in particular $(n, p)$ ), where experiments so suggested.

An example is shown in Fig. 10, where the computed response function for a $8.9 \mathrm{MeV}$ monocromatic 


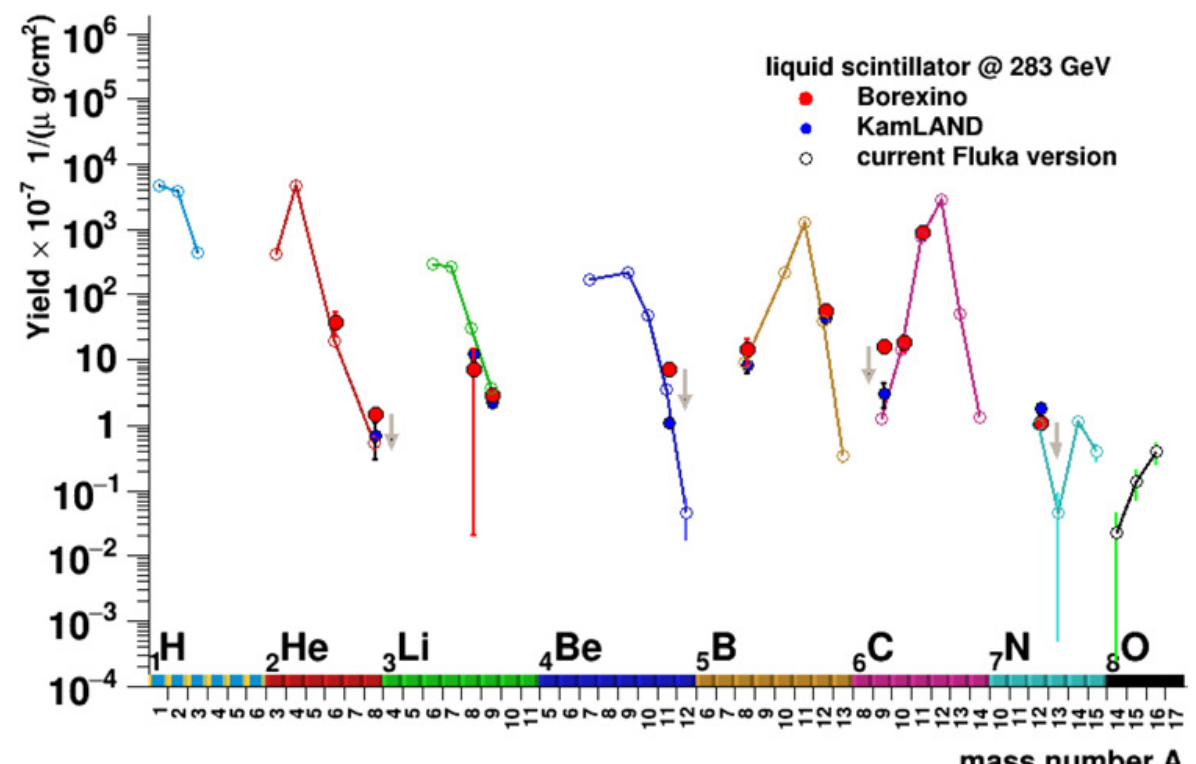

Figure 5. Muon-induced cosmogenic isotope production yields in liquid scintillator underground detectors, assuming realistic muon spectra with $283 \mathrm{GeV}$ average energy. Experimental results from Borexino [22] and KamLAND [23] are shown by the red and blue symbols respectively: when present, arrows indicate an upper limit. Preliminary predictions obtained with the current version of FLUKA for the measured isotope production rates are shown by the open symbols.

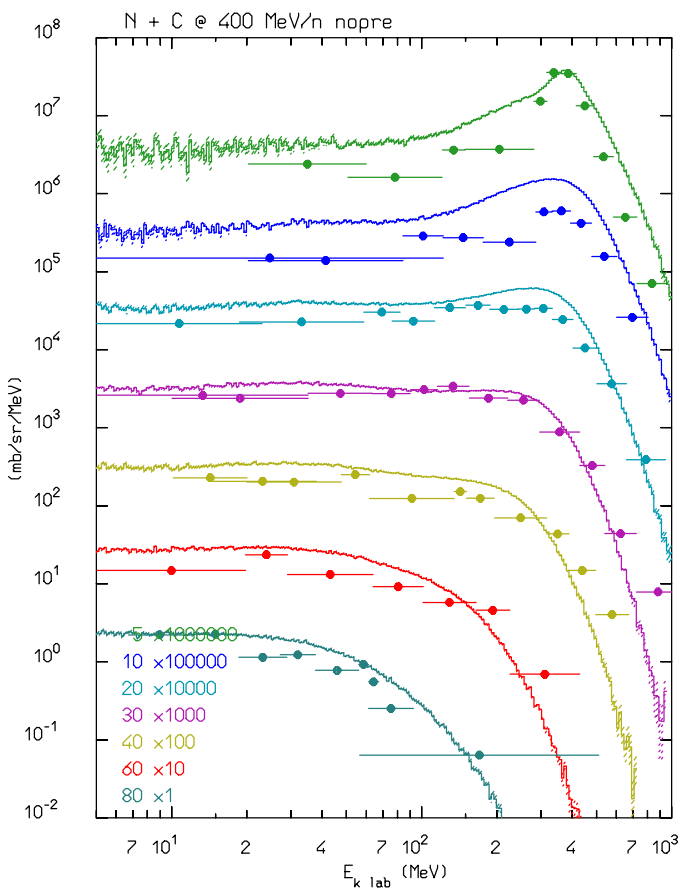

Figure 6. $\mathrm{DDCX} C\left({ }^{14} \mathrm{~N}, \mathrm{xn}\right)$ at $400 \mathrm{MeV} / \mathrm{n}$ as a function of energy for various angles (indicated in figure). Symbols: exp. data [30], histograms: FLUKA with the previous RQMD version.

neutron beam of a BC505 liquid scintillator cell is compared with experimental data [32]. The simulation faithfully implements the experimental multi-cell setup and the associated veto and anticoincidence logic. Signal quenching and detector resolution are also accounted for.

\section{Single Event Effects}

The response of commercial electronic components to Single Event Effects (SEEs) can be calibrated and used as detectors for radiation monitoring.

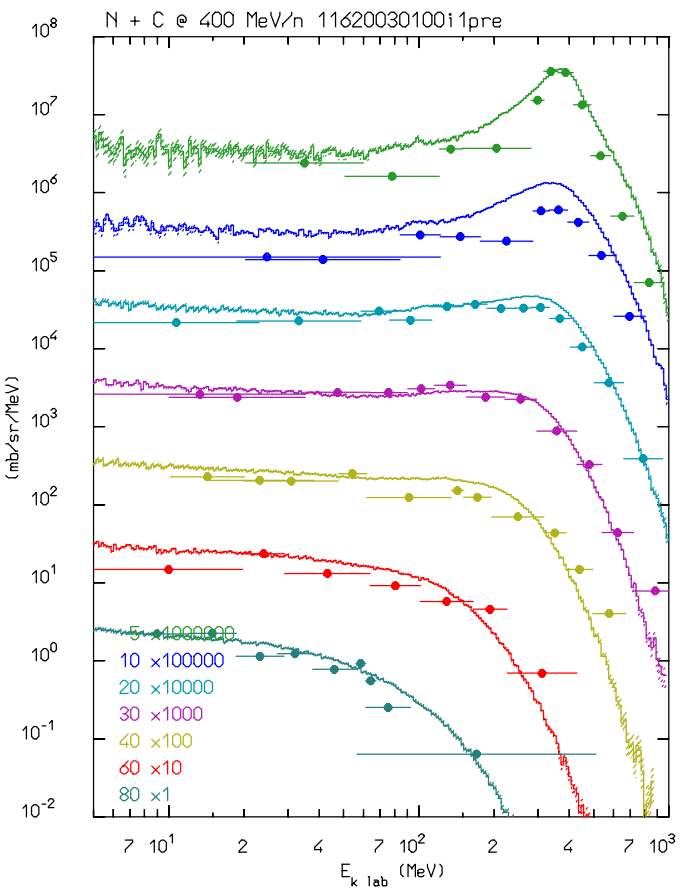

Figure 7. DDCX C $\left({ }^{14} \mathrm{~N}, \mathrm{xn}\right)$ at $400 \mathrm{MeV} / \mathrm{n}$ as a function of energy for various angles (indicated in figure). Symbols: exp. data [30], histograms: FLUKA with the modified RQMD.

The SEE detectors to be used in the high-energy accelerator field are typically calibrated using protons up to $200 \mathrm{MeV}$, neutrons in the 5-15 MeV range and thermal neutrons. Similar approaches apply to other applications such as space (also including heavy ions) or medical.

Once calibrated, the detectors can be used in a mixed field and benchmarked against FLUKA calculations of the radiation field. The agreement for the mixed field in the CHARM test facility [33] at CERN for the hadron 


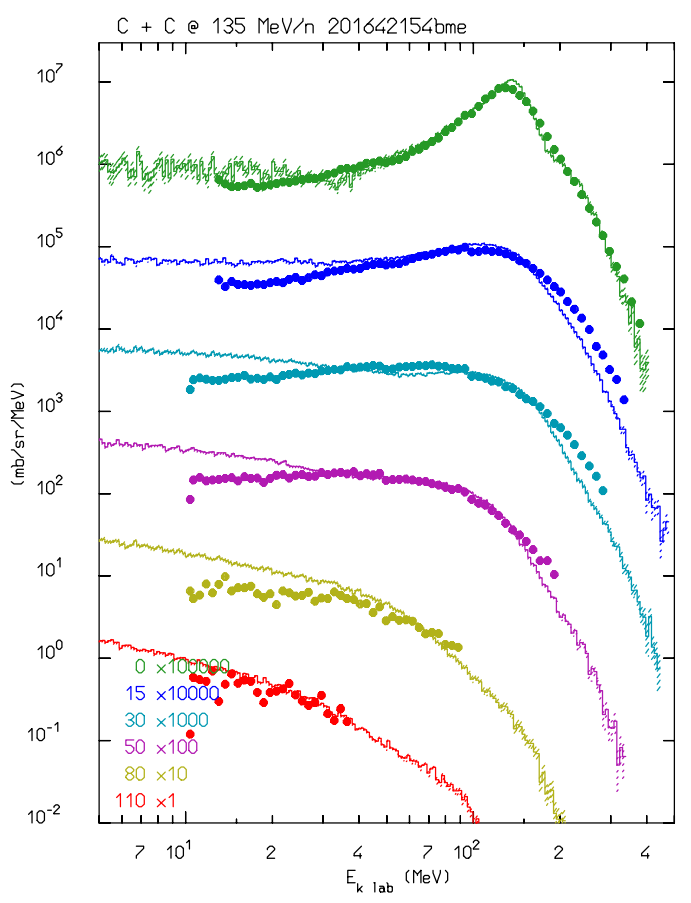

Figure 8. $\mathrm{DDCX} C\left({ }^{12} \mathrm{C}\right.$, xn $)$ at $135 \mathrm{MeV} / \mathrm{n}$ as a function of energy for various angles (indicated in figure). Symbols: exp. data [29], histograms: FLUKA with the modified BME.

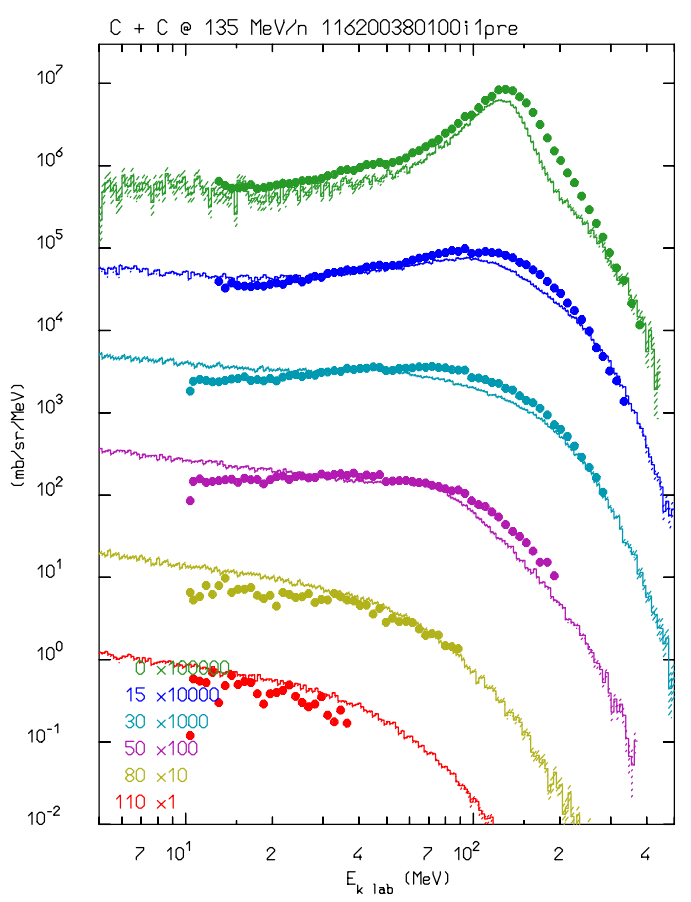

Figure 9. $\mathrm{DDCX} C\left({ }^{12} \mathrm{C}, \mathrm{xn}\right)$ at $135 \mathrm{MeV} / \mathrm{n}$ as a function of energy for various angles (indicated in figure). Symbols: exp. data [29], histograms: FLUKA with the modified RQMD.

above $20 \mathrm{MeV}$ (HEH) fluence measurement is within the uncertainty of the test experimental data [34].

Components with high- $Z$ materials near their sensitive volume (e.g. plugs and connectors in SRAM memories) and high LET thresholds can have a strong SEE cross section dependence on energy. FLUKA is used to evaluate the impact of the high- $Z$ materials up to energies still relevant for the high-energy accelerator environment (e.g. $\mathrm{GeV}$ range) but not accessible in standard cyclotron test

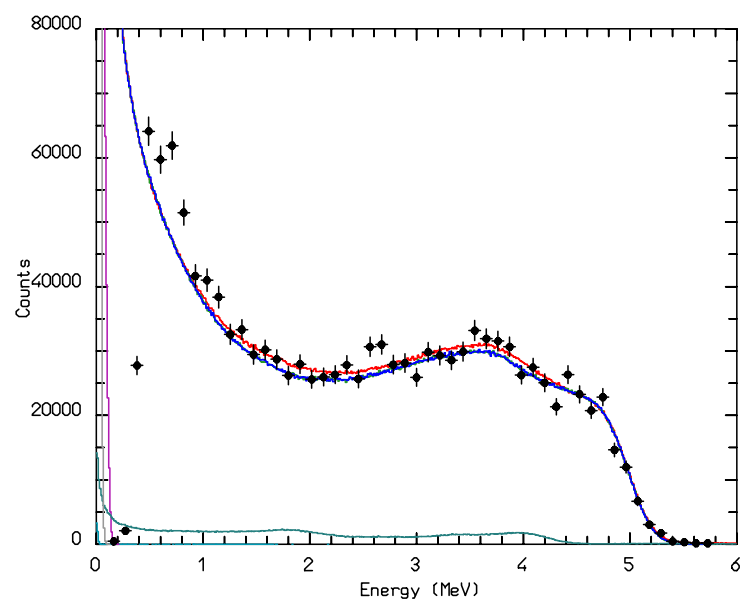

Figure 10. Pulse height spectra for a BC505 liquid scintillator cell with $8.9 \mathrm{MeV}$ incident neutrons. Black symbols: exp. data [32], colored curves: simulated response accounting for quenching and energy resolution. Red curve: total response, blue: proton contribution, light blue: deuterons, purple: alphas, dark green: electrons, grey: heavy recoils.

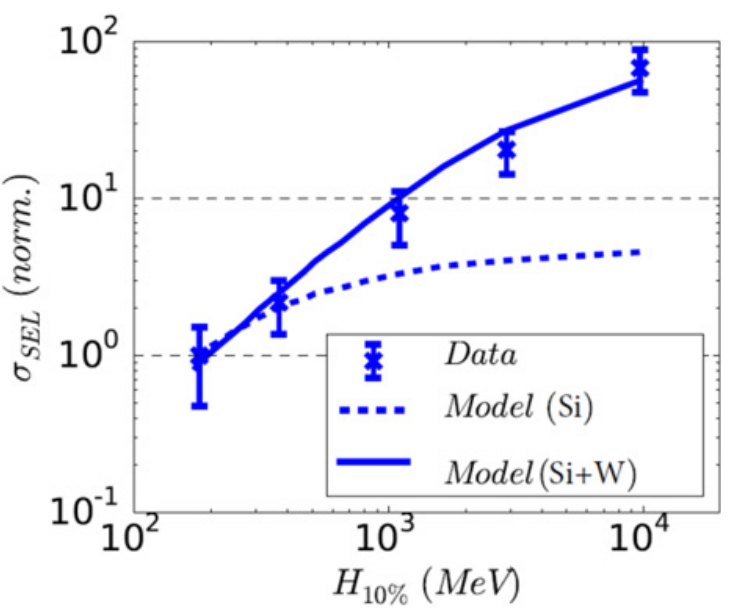

Figure 11. Single Event Effect cross section as measured at CHARM (symbols) and predicted by FLUKA without and with heavy materials, as a function of the spectral hardness.

facilities. If silicon (or silicon-like) materials dominate, the SEE cross section has a weak dependence with energy above several tens of $\mathrm{MeV}$, however even small amounts of e.g. tungsten can lead to a strong energy dependence.

A FLUKA model including the high- $Z$ material has been used to calculate the SEE cross section in the CHARM mixed field as a function of the spectral hardness $\mathrm{H}_{10 \%}$, defined as the energy above which $10 \%$ of the HEH spectrum remains. For components with high- $Z$ material dominated cross sections, testing at typical cyclotron energies $(50-200 \mathrm{MeV})$ can lead to a significant underestimation of the SEE rates in high-energy accelerator environments, as demonstrated in Fig. 11 with data taken at CHARM for a specific chip.

\section{Conclusions}

Nuclear models in the FLUKA code have been further improved to match new challenges at high and low energy frontiers. Comparisons with thin target experimental data demonstrate the quality of the models. Examples 
of applications range from medical physics to future accelerator design, including delicate "environments" such as underground neutrino detectors and radiation damage as a mixed field monitoring tool.

\section{References}

[1] A. Ferrari, P.R. Sala, A. Fassò, J. Ranft, "FLUKA: a multi-particle transport code", CERN-2005-10 (2005), INFN/TC_05/11, SLAC-R-773

[2] T.T. Böhlen et al., Nuclear Data Sheets 120, 211 (2014)

[3] G. Battistoni et al., Annals of Nuclear Energy 82, 10 (2015)

[4] S. Roesler, R. Engel and J. Ranft (2001), Proceedings of the Monte Carlo 2000 Conference, Lisbon, October 23-26 2000, A. Kling, F. Barão, M. Nakagawa, L. Távora, P. Vaz eds., Springer-Verlag Berlin, p. 1033

[5] R. Engel, Z. Physik C66, 203 (1995)

[6] R. Engel and J. Ranft, Phys. Rev. D54, 4244 (1996)

[7] A. Fedynitch, R. Engel, Proc. 14th Int. Conf. on Nuclear Reaction Mechanisms, Varenna, Italy, 1519 June 2015, CERN-Proceedings-2015-001, 291 (2015)

[8] The LHCb Collaboration, Eur. Phys. J. C74, 2888 (2014)

[9] H.H. Braun, A. Fassò, A. Ferrari, J.M. Jowett, P.R. Sala, G.I. Smirnov, Phys. Rev. ST Accel. Beams 17, 021006 (2014)

[10] C. Bertulani, and C. Baur, Phys. Rep. 163, 299 (1988)

[11] C.W. Soto Vargas, D.S. Onley, and L.E. Wright, Nucl. Phys. A288, 45 (1977)

[12] F. Zamai-Noor, and D.S. Onley, Phys. Rev. C33, 1354 (1986)

[13] P. Durgapal, and D.S. Onley, Phys. Rev. C27, 523 (1983)
[14] P. Durgapal, and D.S. Onley, Comp. Phys. Comm. 32, 291 (1984)

[15] V.M. Budnev, I.F. Ginzburg, G.V. Meledin, and V.G. Serbo, Phys. Rep. 15, 181 (1975)

[16] E. Wolynec, V.A. Serrao, and M.N. Martins, J. Phys. G: Nucl. Phys. 13, 515 (1987)

[17] M.L. Justice et al., Phys. Rev. C49, R5 (1994)

[18] J. Aschenbach, and R. Haag, Z. Physik A292, 285 (1979)

[19] J.D.T. Arruda Neto, S.B. Herdade, B.S. Bhandari, and I.C. Nascimento, Phys. Rev. C14, 1499 (1976)

[20] H. Ströher et al., Nucl. Phys. A378, 237 (1982)

[21] A. Empl, A. Ferrari, E.V. Hungerford, and G.I. Smirnov, AIP Conf. Proc. 1672, 090001 (2015)

[22] G. Bellini et al., J. Cosm. Astr. Phys. 2013, 049 (2013)

[23] K. Eguchi et al., Phys. Rev. Lett. 90, 021802 (2003)

[24] V. Andersen et al., Adv. Space Res. 34, 1302 (2004)

[25] H. Sorge, H. Stöcker, and W. Greiner, Ann. Phys. (N.Y.) 192, 266 (1989)

[26] H. Sorge, Phys. Rev. C52, 3291 (1995)

[27] F. Cerutti et al., Proc. 11th Int. Conf. on Nuclear Reaction Mechanisms, Varenna, Italy, 12-16 June 2006, E. Gadioli ed., 507 (2006)

[28] G. Battistoni et al., Frontiers in Oncology, Radiation Oncology Section, 00116 (2016)

[29] H. Sato et al., Phys. Rev. C64, 034607 (2001)

[30] C. Heilbronn et al., Nucl. Sci. Eng. 157, 142 (2007)

[31] T. Ogawa, T Sato, S. Hashimoto, and K. Niita, Nucl. Instr. Meth. A763, 575 (2014)

[32] E. Korkmaz et al., Nucl. Instr. Meth. A431, 446 (1999)

[33] J. Mekki, et al., IEEE Trans. Nucl. Sci. 63, 2106 (2016)

[34] R. Garcia Alia et al., IEEE Trans. Nucl. Sci. 62, 2555 (2015) 